\title{
Empirical Assessment of Breast Lesion Detection Capability Through an Innovative Microwave Imaging Device
}

\author{
Lorenzo Sani*, Alessandro Vispa*, Navid Ghavami*, Riccardo Loretoni ${ }^{\dagger}$, Michele Duranti ${ }^{\ddagger}$, \\ Daniel Alvarez Sánchez-Bayuela*, Stefano Caschera*, Martina Paoli*, Alessandra Bigotti*, \\ Mario Badia*, Michele Scorsipa*, Giovanni Raspa*, Mohammad Ghavami ${ }^{\S}$, Gianluigi Tiberi ${ }^{*}$ \\ ${ }^{*}$ UBT - Umbria Bioengineering Technologies, Perugia, Italy, \\ ${ }^{\dagger}$ Breast Unit, Foligno Hospital, Foligno, Italy, \\ \#-pepartment of Diagnostic Imaging, Perugia Hospital, Perugia, Italy, \\ ${ }^{\S}$ School of Engineering, London South Bank University, London, UK, Email: tiberig@1sbu.ac.uk
}

\begin{abstract}
This paper investigates the effect of conductivity weighting on microwave images obtained through a dedicated imaging device. MammoWave is a microwave imaging device for detection of breast lesions, operating using only two azimuthally rotating antennas without the use of matching liquids. For each breast, a set of conductivity weighted images are generated through modifying our algorithm based on Huygens principle, producing intensity maps representing the homogeneity of tissues' dielectric properties. Subsequently, we introduce several imaging parameters (i.e. features) to quantify the non-homogenous behavior of the image. Through empirical investigation on 103 breasts, we can verify that a selection of these features could allow distinction between breasts with radiological findings (WF), i.e. with benign or malign lesions, and breasts with no radiological findings (NF). Statistical significance was set at $\mathrm{p}<\mathbf{0 . 0 5}$. We obtained single features Area Under the receiver operating characteristic Curves (AUCs) spanning from 0.65 to 0.68 . Significantly, we achieve AUCs of up to 0.77 when considering dense breasts only, which tend to cause detection limitations in mammography exams.
\end{abstract}

Index Terms-Huygens principle, Microwave imaging, Breast cancer detection.

\section{INTRODUCTION}

Mammography has long been established as the gold standard technology for breast screening to reduce breast cancer mortality [1-3]. However, it comes with drawbacks and safety concerns, including the use of ionizing radiation, breast compression and performance restrictions due to x-rays' intrinsic nature. Significantly, breast density is a restrictive property that can prevent breast cancer detection in mammograms of women with radiographically dense breasts $[4,5]$. In general, women are eligible for biannual screening after the age of 49 in order to minimize the impact of ionizing radiation. However, recent studies estimate that breast cancer is diagnosed in $6.6 \%$ of women below the age of 40 [4], and an average of $20 \%$ of breast cancer cases in Europe occur in females younger than 50 years old [6].

Many efforts are underway to develop non-ionizing technologies which could enable screening without age or followup examination interval restrictions. Among these, microwave imaging has appeared as a promising technology for breast lesions detection [7]. Microwave imaging methods aim to discriminate between healthy tissues and tissues with lesions by exploiting their contrast in dielectric properties, i.e. permittivity and conductivity, within microwave frequencies. A high contrast (up to 5) has been reported [7] between healthy breast tissue and malignant tissue, while newer studies confirm a high contrast only between malignant and fatty breast tissues, and less contrast between malignant and healthy fibro glandular tissues [8, 9].

Microwave imaging techniques may be separated into two main groups: microwave tomography and ultra-wideband (UWB) radar methods [10]. Microwave tomography is based on inverse scattering algorithms that create maps of permittivity and conductivity; however, inverse scattering approaches could suffer from mathematical instability, which may not converge to a meaningful solution. UWB radar methods instead perform a linear reconstruction of the image, which is a scattering map in arbitrary units. A number of microwave imaging prototypes with varying hardware and imaging algorithms have been constructed and reached clinical level testing [11].

As one of the few clinically ready products, MammoWave operates with only two azimuthally rotating antennas without using any matching liquids. MammoWave has an innovative frequency domain imaging algorithm, based on Huygens Principle (HP) [12]. The device has previously been presented, tested and clinically validated [13-15]. The apparatus produces images in the form of intensity maps, representing the homogeneity of tissues' dielectric properties. In this paper, for each breast we generate a set of conductivity weighted microwave images. Next, several image features are calculated to quantify and measure the non-homogenous behaviour of the image. We show that an appropriate selection of these features could allow distinction between breasts with radiological findings (WF), and breasts with no radiological findings (NF). We have empirically verified the procedure on 103 breasts, each 
one with the correspondent radiologist review output obtained using MRI and/or mammography and/or echography.

The outline of this paper is as follows. Section II describes the apparatus and the imaging algorithm based on HP. Feature extraction and feature selection procedures are also explained in this section. Results are presented and discussed in section III, while section IV concludes the work and highlights the future work.

\section{MATERIALS AND Methods}

\section{A. Microwave apparatus and imaging algorithm}

The MammoWave system (fig. 1(a)), consists of an aluminum cylindrical hub containing two antennas, one transmitting (tx) and one receiving antenna (rx), which operate from 1 to $9 \mathrm{GHz}$. The hub is internally covered by microwave absorbers, and is equipped with a hole with a cup, allowing the insertion of the patient's breast, with the patient lying in a prone position (fig. 1(b)). The antennas are installed at the same height, in free space and rotate around the azimuth collecting the microwave signals from different angular positions [13]. The tx and rx are connected to a 2-port VNA (Cobalt C1209, Copper Mountain) which operates up to $9 \mathrm{GHz}$.

Measurements were performed recording the complex S21 in a multi-bistatic fashion, such that for each transmitting position $\mathrm{tx}_{m}$ the receiving antenna is moved to measure the received signal every $4.5^{\circ}$, resulting in 80 receiving points $\mathrm{rx}_{n p}$. A total of 10 transmitting positions have been used in all measurements, displaced in 5 sections centered at $0^{\circ}$, $72^{\circ}, 144^{\circ}, 216^{\circ}$, and $288^{\circ}$. Fig. 1(c) illustrates the set-up configuration. In each section the transmitting positions may be displaced by $9^{\circ}$. For each tx and rx position, the complex S21 is collected from 1 to $9 \mathrm{GHz}$, with $5 \mathrm{MHz}$ sampling.

Assuming that $\mathrm{rx}$ can be rotated to measure the received signal at the points $\mathrm{rx}_{n p} \equiv\left(a_{0}, \phi_{n p}\right) \equiv \vec{\rho}_{n p}$ displaced along a circular surface having radius $a_{0}$, the received signals can be expressed as $\mathrm{S} 21_{n}^{m, p}\left(a_{0}, \phi_{n} ; \mathrm{tx}_{m, p} ; f\right)$, where $n=1,2, \ldots, 80$, and $m=1,2 \ldots, 5$ indicate the receiving points and the transmitting sections, respectively, $p=1,2$ and $p^{\prime}=1,2$ indicate the position inside each transmitting section, and $f$ is the frequency. The received signals are then processed through HP to calculate the field inside the cylinder, which is then used to generate an image. To remove the artefacts [16], we employ the subtraction between S21 obtained using two measurements belonging to the doublet of the same section:

$$
\begin{aligned}
& E_{\mathrm{HP}, 2 \mathrm{D}}^{\mathrm{rcstr}}\left(\rho, \phi ; \mathrm{tx}_{m, p}-\mathrm{tx}_{m, p^{\prime}} ; f\right) \\
& \propto \sum_{n=1}^{N_{\mathrm{PT}}}\left(\mathrm{S} 21_{n}^{m, p}-\mathrm{S} 21_{n}^{m, p^{\prime}}\right) G\left(k_{1}\left|\vec{\rho}_{n}-\vec{\rho}\right|\right)
\end{aligned}
$$

where $(\rho, \phi) \equiv \vec{\rho}$ is the observation point, and $\Delta s$ is the spatial sampling. Wave number is indicated by $k_{1}$, and $G$ represents the Green's function. The reconstructed internal field has been indicated by the string "rcstr" while the string HP indicates that Huygens based procedure will be employed in (1). If the conductivity of the media is not equal to zero,

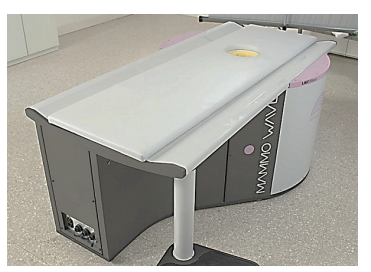

(a)

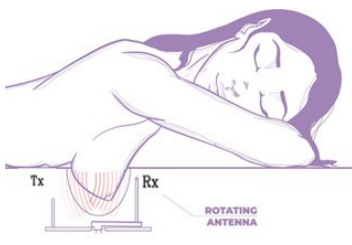

(b)

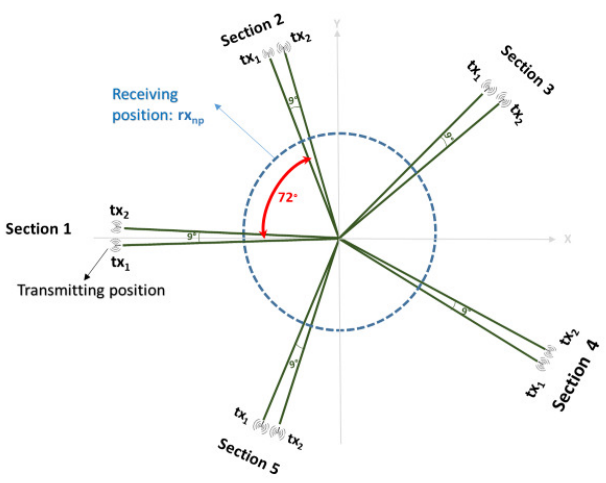

(c)

Fig. 1. The MammoWave system (a). The hub is equipped with a hole with a cup, allowing the insertion of the patient's breast in a prone position (b). The antennas are installed at the same height, in free space and rotate around the azimuth to collect the signals from different angular positions (c).

(1) compensates the attenuation experienced when going into the media. Assuming we use $N_{F}$ frequencies $f_{i}$ in the band $B$, the intensity of the image $I$ can be obtained using (2) through incoherent summation of all the solutions from all sections:

$$
I(\rho, \phi)=\sum_{m=1}^{5} \sum_{\substack{p=1 \\ p^{\prime}=1 \\ p \neq p^{\prime}}}^{2} \sum_{i=1}^{N_{F}}\left|E_{\mathrm{HP}, 2 \mathrm{D}}^{\mathrm{rcstr}}\left(\rho, \phi ; \mathrm{tx}_{m, p}-\mathrm{tx}_{m, p^{\prime}} ; f_{i}\right)\right|^{2}
$$

\section{B. In-vivo validation}

MammoWave's in-vivo validation on volunteers in Perugia and Foligno Hospitals was approved in 2015 by the Ethical Committee of Umbria, Italy (N. 6845/15/AV/DM of $14 / 10 / 2015, \quad$ N. 10352/17/NCAV of 16/03/2017, N 13203/18/NCAV of 17/04/2018), for a feasibility study on detection of breast lesions with the aim of quantifying its potential for use in medical technology screening. The inclusion criteria allowed female volunteers above 18 years old with intact breast skin and with a radiologist study output obtained through conventional exams (mammography and/or ultrasound and/or MRI) within the last month. All protocols and procedures were in accordance with both institutional and national ethical standards in research, and with World Medical Association Declaration of Helsinki (1964) and its later amendments or analogous ethical standards. Prior to the trial, all participants have been requested to read and sign both the informative sheet and informed consent form.

The results obtained using the data from 103 breasts are presented here. Each breast has its own correspondent output 
of the radiologist study review, for use as gold standard for classification of the breasts into two categories: those with no radiological finding (NF), and those with radiological findings (WF). Moreover, where possible the breast type has been classified according to its density, following the scale defined by the American College of Radiology (ACR) ranked from ACR1 (extremely fatty breast) to ACR4 (extremely heterogeneous fibroglandular breast) [16]. Specifically, we will denote ACR3 and ACR4 breasts as dense. The lesion type includes fibroadenoma, carcinoma, nodule, microcalcifications, cyst and other lesions [17-19].

After agreeing to participate, the subject is assisted by the clinical study coordinator; she positions her breast in the cup, which is appropriately integrated in a bed as shown in fig. 1(b). Three biocompatible cups made of polylactic acid [20] having varying sizes are available, and the clinical study coordinator chooses the one that better fits the subject's breast. The thickness of the cup is only $1 \mathrm{~mm}$, and does not impact the imaging results [14]. It should be emphasized that no matching liquid is used in the apparatus, and no breast compression has to be applied during acquisition.

All microwave images have been obtained in a cylindrical grid with a radius of $7 \mathrm{~cm}$ (rotation radius of $\mathrm{rx}$ ), a radial sampling of $1 \mathrm{~mm}$ and an azimuthal sampling of $3^{\circ}$. The images have been obtained using free space dielectric constant in (1), due to the position of $\mathrm{rx}$ in air. For each breast we investigated the variation of conductivity $(\sigma)$ from 0 to $0.9 \mathrm{~S} / \mathrm{m}$ (0.1 S/m sampling). When applying (1). We will refer to such microwave images as conductivity weighted microwave images, denoted as $\mathrm{MI}_{\sigma}$. Images obtained here are intensity maps, given in linear arbitrary units, representing the homogeneity of tissues' dielectric properties. To allow inter and intra-subject comparison, all images are normalized to unitary average of the intensity.

\section{Feature Extraction}

To quantify the non-homogenous behaviour of the microwave images, we introduce the following features:

- $\mathrm{MIN}=$ Minimum image intensity;

- MAX = Maximum image intensity;

- MEA = Mean value of image intensity;

- $\mathrm{MED}=$ Median value of image intensity;

- VAR = Variance of image intensity;

- MAD0 = Mean absolute deviation of image intensity;

- MAD1 = Median absolute deviation of image intensity;

- KUR = Kurtosis of the image;

- $\mathrm{SKE}=$ Skewness of the image;

- $\mathrm{M} 2 \mathrm{AVG}=(\mathrm{MAX}) /(\mathrm{MEA})$;

- $\mathrm{ROS1}=(\mathrm{MAX}-\mathrm{MIN}) /(\mathrm{MEA}-\mathrm{MIN})$;

- $\operatorname{ROS} 2=($ MAX-MIN) $/($ MED-MIN);

- $\mathrm{ENT}=$ Entropy of the image.

For each conductivity weighted image, these are calculated on the full image domain, i.e. feature $\left[\mathrm{MI}_{\sigma}^{\text {full image }}\right]$, denoted with the subscript " $i$ ". Additionally, for each conductivity weighted image, all features excluding KUR, SKE, ROS1,
ROS2 are calculated on the peak region (a region which is centered in the maximum of the image and it extends to MAX/2), denoted with the subscript " $p$ "; and on its complementary, denoted with the subscript " $c$ ". The ratios between features calculated on the peak region and on its complementary are considered as added features, and are highlighted with subscript " $r$ ".

Next, for each feature, using the gold standard output of the radiologist study review (NF or WF category), we calculate: the mean and standard deviation for both NF and WF breasts. In addition, for each feature, using the radiologist study review output, Welch's t-test (i.e. a two-sample two-tailed unpooled variances t-test) with $\alpha=0.05$ has been performed. Statistical significance was set at $\mathrm{p}<0.05$. We also numerically evaluated the receiver operating characteristic (ROC): specifically, we evaluated True Positive (TP) and False Negative (FN) rates for each feature of each conductivity weighted image. In more details, since TP rate and FN rate depend on the classifier threshold, i.e. the decision offset, we empirically calculated ROC curves by adjusting the decision offset and calculating $\mathrm{TP}$ and FN for all possible decision offsets. Thus, the area under the curve (AUC) is determined.

\section{Feature Selection and Calculations}

With the aim of empirically verifying if an appropriate selection of microwave image features may allow discriminating between NF and WF breasts, the following steps are performed for each conductivity weighted image: i) for the ROC of each feature, the TP rate obtained for True Negative (TN) rate $\mathrm{TN}=0.55$, i.e. $\left.\mathrm{TP}\right|_{\mathrm{TN}=0.55}$ is calculated, and the corresponding decision offset is annotated. ii) we order the feature with decreasing $\left.\mathrm{TP}\right|_{\mathrm{TN}=0.55}$ and we select the first four after checking that $\mathrm{p}<0.05$ is verified.

\section{RESULTS AND DISCUSSIONS}

According to the radiologist study review, a total number of $52 \mathrm{NF}$ (19 dense) and $51 \mathrm{WF}$ ( 22 dense) breasts were analyzed, which included nodules, cyst, carcinoma, fibroadenoma, microcalcifications.

Four selected features corresponding to three different conductivity weighed images are listed in Table 1. For each feature, we indicate: the mean and standard deviation for the NF breasts, the mean and standard deviation for the WF breasts, the decision offset corresponding to $\mathrm{TN}=0.55$, the AUC for all breasts and AUC for dense breasts only. ROC curves (empirically calculated) are shown in Fig. 2.

Two breasts are shown here in more details as test cases, each one with three of the selected conductivity weighed microwave images (for $\sigma$ equal to $0.3 \mathrm{~S} / \mathrm{m}, 0.6 \mathrm{~S} / \mathrm{m}$ and 0.8 $\mathrm{S} / \mathrm{m}$, respectively). Microwave images, normalized to unitary average of the intensity, are given here as $2 \mathrm{D}$ images in the azimuthal (coronal) plane and are divided into four quadrants corresponding to breast Upper-Outer (UO) quadrant; UpperInner (UI) quadrant; Lower-Outer (LO) quadrant; Lower-Inner (LI) quadrant. Moreover, 1D intensity projection on $\mathrm{x}$ and $\mathrm{y}$ is displayed in the inserts. $\mathrm{X}$ and $\mathrm{y}$ axes are in meters 
TABLE I

LIST OF THE SELECTED FEATURES FOR 3 CONDUCTIVITY WEIGHED IMAGES

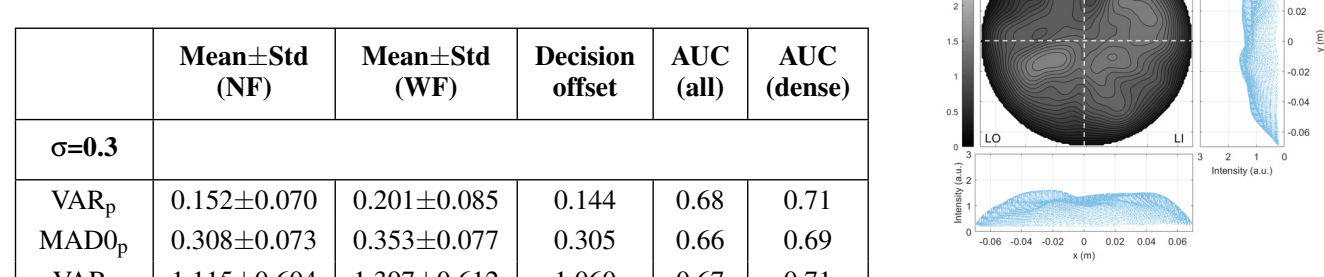

(a)

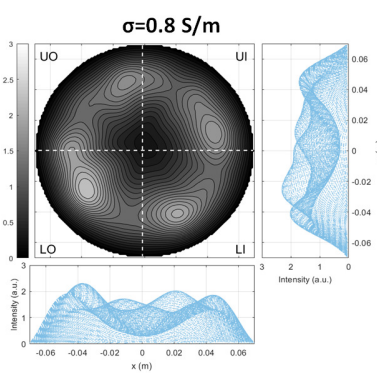

(c)

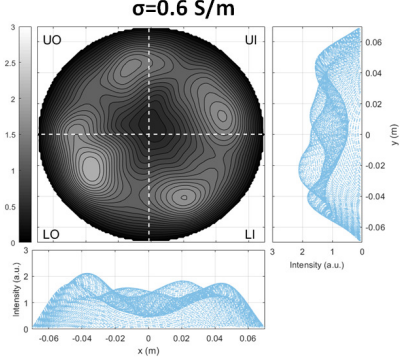

(b)
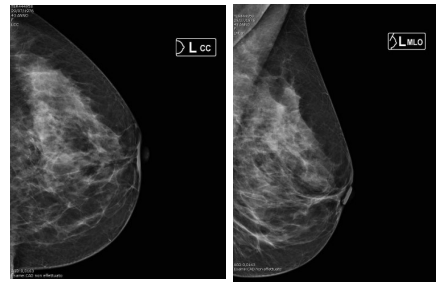

(d)

\begin{tabular}{l|l|l|l|l|l} 
ROS $_{\mathrm{i}}$ & $2.731 \pm 0.423$ & $3.013 \pm 0.494$ & 2.674 & 0.67 & 0.76
\end{tabular}

\begin{tabular}{l|l|l|l|l|l|} 
ROS2 & $2.908 \pm 0.708$ & $3.346 \pm 0.857$ & 2.773 & 0.66 & 0.71 \\
\hline
\end{tabular}

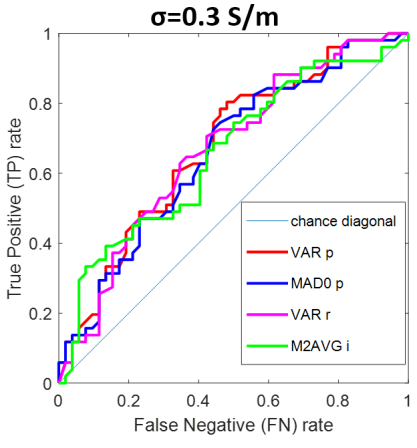

(a)

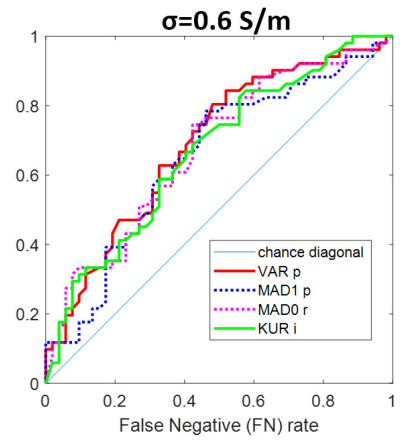

(b)

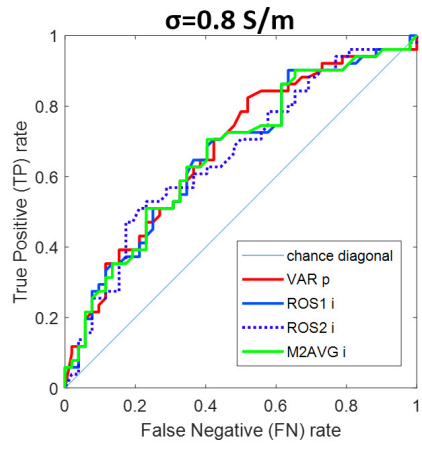

(c)

Fig. 2. ROC curves of the selected features for $\sigma$ values equal to 0.3 (a), 0.6 (b), and 0.8 (c).

while intensity is in arbitrary units. For each test case the output and main findings of the radiologist study review, with the correspondent conventional images, is also given. Fig. 3 refers to a NF breast, while Fig. 4 refers to a WF breast with microcalcification.
Fig. 3. NF heterogeneously dense (ACR 3) breast; (a)-(c) represent the microwave images (in arbitrary units) when employing $\sigma$ values $0.3,0.6$, and $0.8 \mathrm{~S} / \mathrm{m}$, respectively. Microwave images (x and y axes are in meters) show a homogeneous behavior. The radiologist study review has been obtained with mammography images (d).

MammoWave does not use any patient-specific estimation, which means that breast images are generated without any prior knowledge of patient-specific breast dielectric properties. In more details, the images have been obtained using free space dielectric constant in (1). Concerning the conductivity, for each breast we varied the conductivity from 0 to $0.9 \mathrm{~S} / \mathrm{m}$ (in agreement with the breast conductivity average values reported in [10]). Here we presented microwaves images corresponding to 3 sample conductivity values within this range.

From visual inspection of microwave images, it can be pointed out that microwave images of WF breasts have a more non-homogenous behaviour with respect to NF breast. This confirms what was previously highlighted in [13, 14], using phantom measurements. Interestingly, small microcalcifications $(1.6 \mathrm{~mm})$ also lead to non-homogenous behaviour which can be visually appreciated.

With the aim of discriminating between WF and NF breasts, some dedicated features have been introduced and selected. Such features allow a quantification of the non-homogeneity of the microwave images: some of them describe the entire image [13, 14], while others describe the peak region [21]. AUC of selected features span from 0.65 to 0.68 . Significantly, when calculating the AUCs of the selected features for dense breasts only, we see an increase of up to 0.77 .

\section{CONCLUSIONS AND FUTURE WORK}

This paper presented an investigation on the effect of conductivity weighting on detection capabilities of a microwave imaging prototype for breast lesion detection. Our 


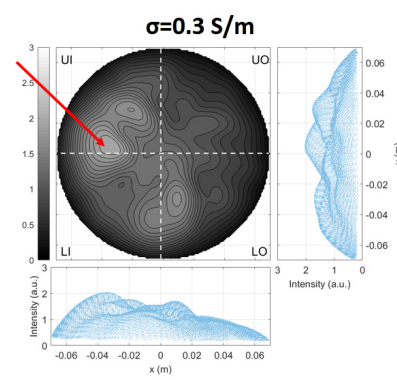

(a)

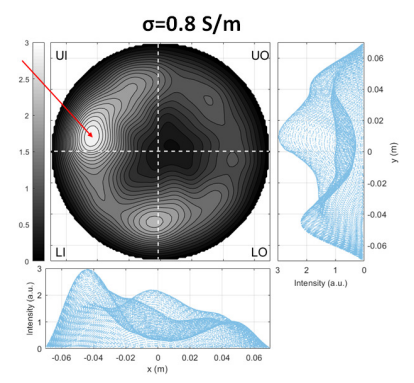

(c)

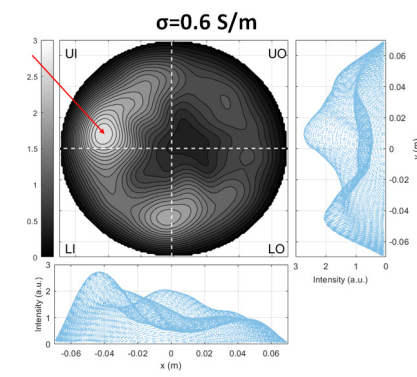

(b)

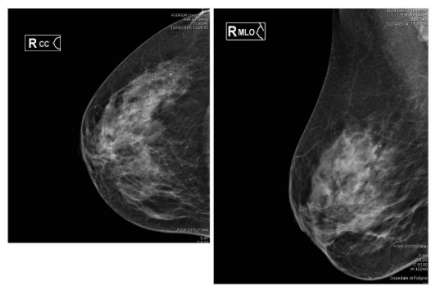

(d)
Fig. 4. WF heterogeneously dense (ACR 3) breast; (a)-(c) represent the microwave images (in arbitrary units) when employing $\sigma$ values $0.3,0.6$, and $0.8 \mathrm{~S} / \mathrm{m}$, respectively. All microwave images (x and y axes are in meters) show a non-homogeneous behavior, with a main peak indicated by the red arrows. The radiologist study review has been obtained with mammography images (d), giving as output the presence of Microcalcifications of $1.6 \mathrm{~mm}$.

results verify that a selection of features obtained from a range of conductivity weighted microwave images may allow discrimination between NF and WF breasts, while passing the Welch's t-test. Next steps will be focused on verifying if an appropriate combination and use of microwave image features may enhance performance accuracy compared with single feature. Further work on MammoWave, which has recently received CE Mark is ongoing and larger clinical trials are planned with the aim of improving clinical evidence on the use of microwave imaging in the breast screening pathway.

\section{ACKNOWLEDGMENT}

This project has received funding from the European Union's Horizon 2020 research and innovation programme under grant agreement No 830265. This project leading to this application has received funding from the European Union's Horizon 2020 research and innovation programme under the Marie Sklodowska-Curie grant agreement No. 793449. This project has received funding from the European Union's Horizon 2020 research and innovation programme under the Marie Sklodowska-Curie grant agreement No. 872752.

\section{REFERENCES}

[1] T. A. Shah and S. S. Guraya, "Breast cancer screening programs: Review of merits, demerits, and recent recommendations practiced across the world," Journal of microscopy and ultrastructure, vol. 5, no. 2, pp. 59-69, 2017.

[2] T. H. Chen, et. al. "Clarifying the debate on population-based screening for breast cancer with mammography: A systematic review of randomized controlled trials on mammography with Bayesian meta-analysis and causal model". Medicine, vol. 96, no. 3, e5684, 2017.
[3] J. A. Hanley, A. Hannigan, and K. M. O'Brien, "Mortality reductions due to mammography screening: contemporary population-based data," PloS one, vol. 12, no. 12, e0188947, 2017.

[4] H. A. Assi, K. E. Khoury, H. Dbouk, L. E. Khalil, T. H. Mouhieddine, and N. S. El Saghir, "Epidemiology and prognosis of breast cancer in young women," Journal of thoracic disease, vol. 5, Suppl. 1, S2-S8, 2013.

[5] J. E. Joy, E. E. Penhoet, and D. B. Petitti editors,Benefits and Limitations of Mammography. In: Saving Women's Lives: Strategies for Improving Breast Cancer Detection and Diagnosis. Institute of Medicine (US) and National Research Council (US) Committee on New Approaches to Early Detection and Diagnosis of Breast Cancer, Washington (DC): National Academies Press (US), 2005. Available from: https://www.ncbi.nlm.nih.gov/books/NBK22311/

[6] J. Ferlay, M. Ervik, F. Lam, M. Colombet, L. Mery, M. Piñeros, A. Znaor, I. Soerjomataram, and F. Bray, Global Cancer Observatory: Cancer Today, Lyon, France, International Agency for Research on Cancer, 2018.

[7] N. k. Nikolova, "Microwave imaging for breast cancer", IEEE Microwave Magazine, vol. 12, no. 7, pp. 78-94, 2011.

[8] M. Lazebnik, L. McCartney, D. Popovic, C. B. Watkins, M. J. Lindstrom, J. Harter, S. Sewall, A. Magliocco, J. H. Booske, M. Okoniewski, and S. C. Hagness, "A large-scale study of the ultrawideband microwave dielectric properties of normal breast tissue obtained from reduction surgeries," Physics in medicine and biology, vol. 52, no. 10, pp. 2637, 2007.

[9] M. Lazebnik, D. Popovic, L. McCartney, C. B. Watkins, M. J. Lindstrom, J. Harter, S. Sewall, T. Ogilvie, A. Magliocco, T. M. Breslin, et al. A large-scale study of the ultrawideband microwave dielectric properties of normal, benign and malignant breast tissues obtained from cancer surgeries," Physics in Medicine and Biology, vol. 52, no. 20, pp. 6093, 2007.

[10] R. C. Conceição, J. Mohr, and M. O'Halloran, An Introduction to Microwave Imaging for Breast Cancer Detection, Biological and Medical Physics, Biomedical Engineering, Springer, 2016.

[11] M. A. Aldhaeebi, K. Alzoubi, T. S. Almoneef, S. M. Bamatraf, H. Attia, and O. M. Ramahi, "Review of microwaves techniques for breast cancer detection," Sensors, vol. 20, pp. 2390, 2020.

[12] N. Ghavami, G. Tiberi, D. J. Edwards, and A. Monorchio, "UWB microwave imaging of objects with canonical shape," IEEE Transactions on Antennas and Propagation, vol. 60, no. 1, pp. 231-239, 2012.

[13] A. Vispa, L. Sani, M. Paoli, A. Bigotti, G. Raspa, N. Ghavami, S. Caschera, M. Ghavami, M. Duranti, and G. Tiberi, "UWB device for breast microwave imaging: phantom and clinical validations," Measurement, vol. 146, pp. 582-589, 2019.

[14] L. Sani, N. Ghavami, A. Vispa, M. Paoli, G. Raspa, M. Ghavami, F. Sacchetti, E. Vannini, S. Ercolani, A. Saracini, M. Duranti, and G. Tiberi, "Novel microwave apparatus for breast lesions detection: Preliminary clinical results," Biomedical Signal Processing and control, vol. 52, pp. 257-263, 2019.

[15] S. P. Rana, M. Dey, G. Tiberi, L. Sani, A. Vispa, G. Raspa, M. Duranti, M. Ghavami, and S. Dudley, "Machine Learning Approaches for Automated Lesion Detection in Microwave Breast Imaging Clinical Data," Scientific Reports, vol. 9, no. 1, pp. 10510, 2019.

[16] American College of Radiology, ACR practice guideline for the performance of screening and diagnostic mammography, Practice Guidelines and Technical Standards, American College of Radiology, Reston, VA, USA, 2008.

[17] F. A. Tavassoli, P. Devilee, editors, World Health Classification of Tumours. Pathology and Genetics of Tumours of the Breast and Female Genital Organs, IARC press, Lyon, 2003.

[18] S. Lakhani, I. O. Ellis, S. J. Schnitt, et al. editors, WHO Classification of Tumour of the Breast, 4th edition, IARC, Lyon, 2012.

[19] N. Perry, M. Broeders, C. De Wolf, S. Trnberg, R. Holland, and L. von Karsa, European Guidelines for Quality Assurance in Breast Cancer Screening and Diagnosis, 4th edition, European Commission, 2006.

[20] D. Da Silva, M. Kaduri, M. Poley, O. Adir, N. Krinsky, J. ShainskyRoitman, and A. Schroeder, "Biocompatibility, biodegradation and excretion of polylactic acid (PLA) in medical implants and theranostic systems," Chemical Engineering Journal, vol. 340, pp. 9-14, 2018.

[21] D. O'Loughlin, et al, "Sensitivity and specificity estimation using patient-specific microwave imaging in diverse experimental breast phantoms," IEEE Transactions on Medical Imaging, vol. 38, no. 1, pp. 303311, 2019. 